\title{
Time Domain Analysis of Polar Plumes Observed with the LASCO/SOHO Coronagraph
}

\author{
A.Llebaria, J.Boulanger, Y.Boursier \\ Laboratoire d'Astrophysique de Marseille (CNRS) \\ F-13376 BP8 Marseille Cedex12, France
}

\begin{abstract}
The polar regions of the solar corona exhibit during solar minima very faint ray-like structures which apparently outline the lines of polar magnetic field. Usually they are named plumes in the solar coronal literature. In order to understand the spatiotemporal characteristics of these plumes we analyze a sequence of 400 continuous observations covering a period of 3 days. For such analysis we are using Hough transform methods and a multiresolution context. The main result of this work is the fractal structure of the electron density distribution in the coronal hole as well as the fractal characteristics of its temporal evolution. The simulation of sequences of coronal images projected from 3D distributions allows a refinement of the physical parameters.
\end{abstract}

\section{INTRODUCTION}

Polar plumes are observable features of the solar corona in white light (WL). Observed for many years during solar eclipses occurring in epochs of minimal solar activity (3 years over a cycle of 11), they appear like faint radial enhancements over the polar regions. Such features are generated by Thompson diffusion of electrons leaving the solar surface. Thus their global morphology was very soon related to the global structure of the polar magnetic field.

The SOHO spacecraft, launched in December 1995 and operating thereafter continuously at the L2 Lagrange point, offers us the opportunity to observe solar plumes for a long time interval. Observations were carried out by using the white light coronagraph LASCO-C2 on board the spacecraft. A coronagraph is a small telescope with an occultor centered on its field of view in order to hide the bright solar disk and to avoid the direct floodlighting of the objective, which would produce excessive straylight. Solar corona is $\sim 10^{-9}$ dimmer than the Sun disk. The field of view of LASCO-C2 is a large annulus around the solar disk covering the range of 2.5-6.5 $\mathrm{R}_{\odot}$ (solar radii). Regular observations carried out by LASCO-C2 and EIT instruments (EIT is a far-UV imager on board the SOHO spacecraft) have allowed a detailed analysis of plumes. The coronal structure emerging from nearly unipolar magnetic footprints, and detected in the UV at the base of the corona, was found to be preserved as WL plumes throughout the altitude range of 1.1-6.0 $R_{\odot}$ (DeForest et al., 1998 ; DeForest , 2001). Plumes show a super-radial expansion in this range of distances, i.e. they emerge near the pole from a small area known as the coronal hole. They are bent in the 1.-2.5 $\mathrm{R}_{\odot}$ range and show great lateral expansion, filling a large virtual cone. Beyond $2.5 \mathrm{R}_{\odot}$ plumes look like linear structures diverging from a point very near the pole, at a distance of $0.84 \mathrm{R}_{\odot}$ of the center of the solar disk. This point is known as the "divergence point" (it does not have any physical meaning). In the LASCO-C2 field of view the plumes appear as a fan-shaped set of straight rays centered on the divergence point (see Fig. 1).

\section{SINOGRAMS AND TID}

The clear conclusion of early studies on temporal evolution of polar plumes (Lamy et al. 1997) was that they have a short lifetime. This fact ruined the hope of recovering the 3D distribution of electron density on the coronal holes by standard tomographic techniques. This technique 

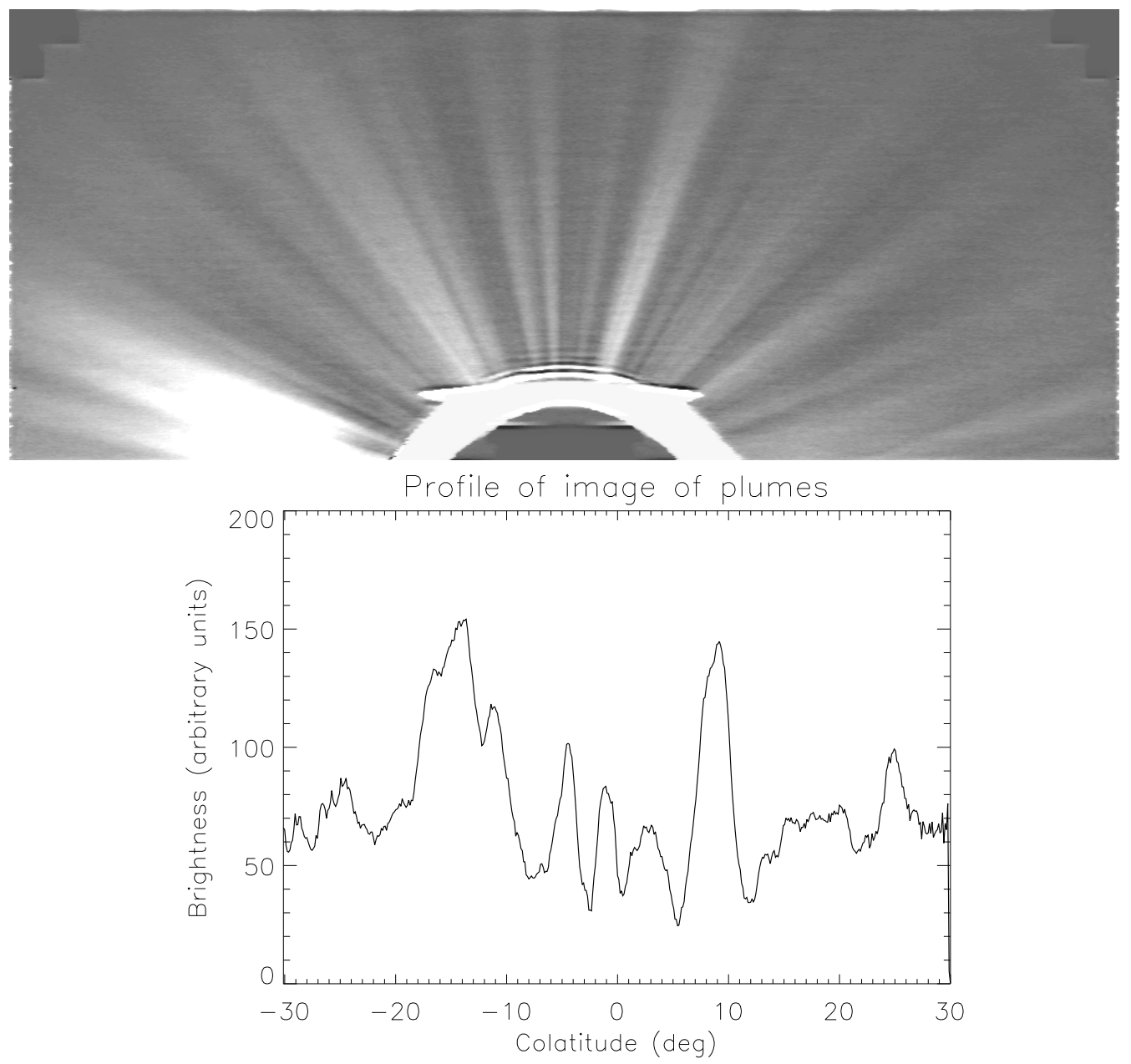

FIGURE 1: Top: A processed image of plumes taken on 24 March 1997 with the LASCO-C2 coronagraph. Bottom: Profile of a circular section of plumes. 

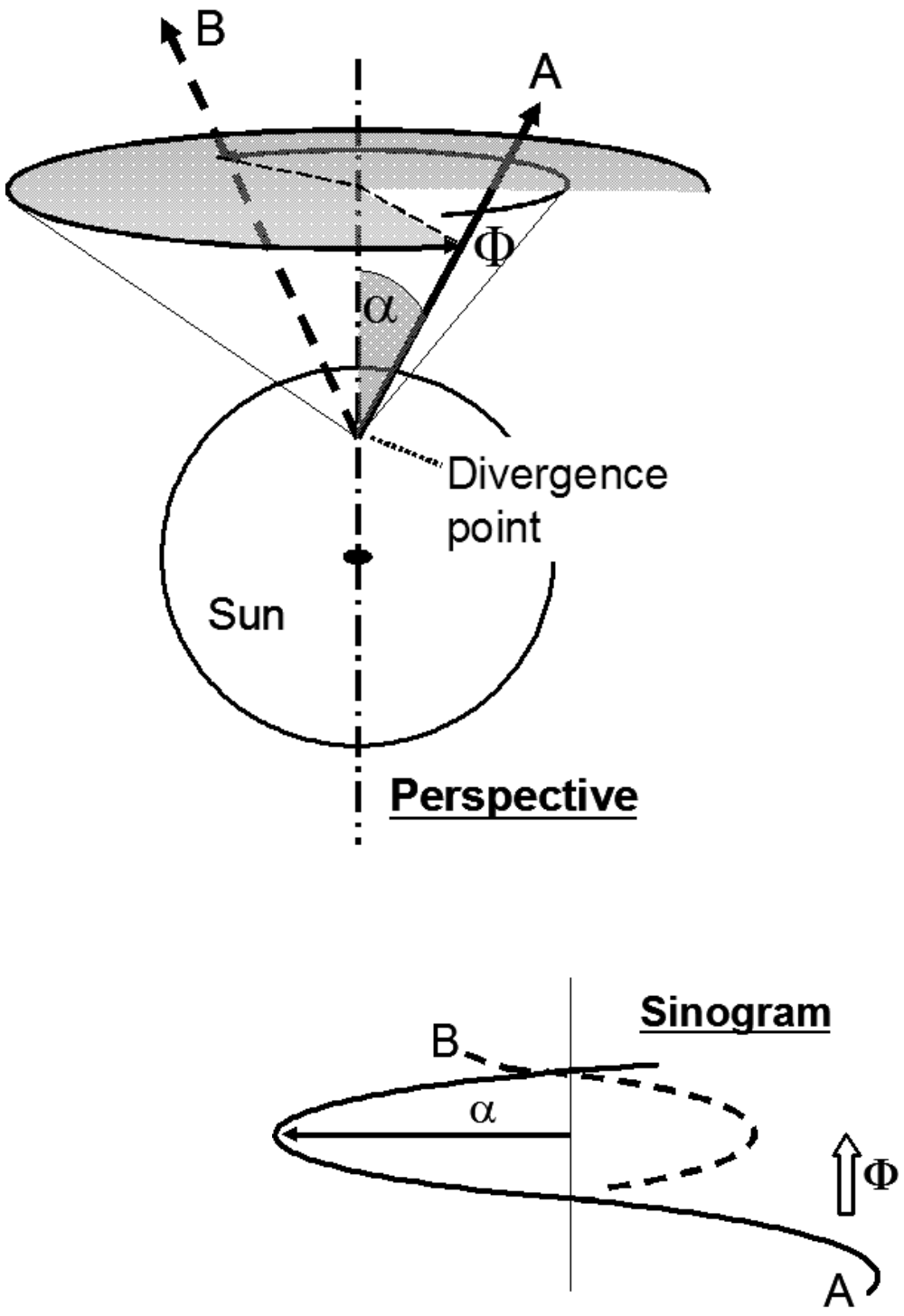

FIGURE 2: Sinogram traces are the graphic representation of plumes rotating arouud the sun solar axis in the plane of coordinates : angle of latitude vs. time. Notice the shifted position of the divergence point. 


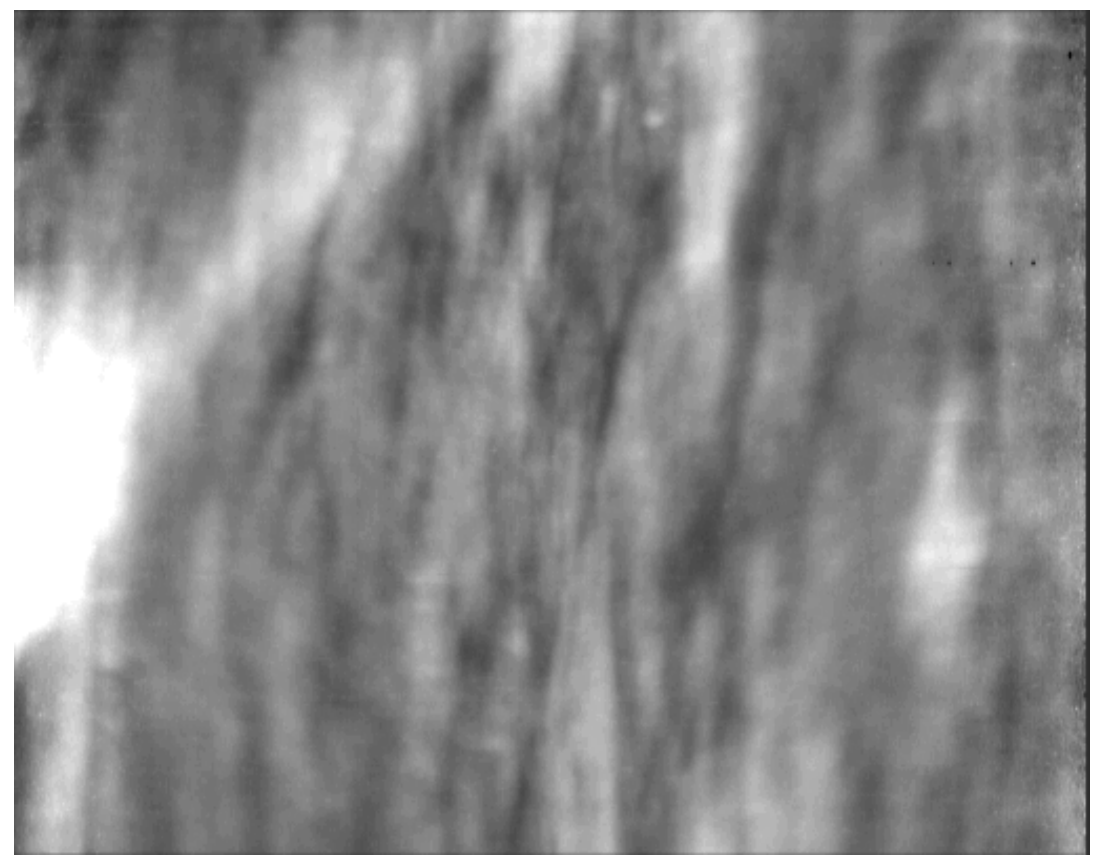

FIGURE 3: TID of the March sequence. The profile at the bottom of Fig. 4 is a row of the upper part of this TID.

uses the sinograms of a stable body to recover a section of it. 2D sinograms depict successive projections of the same section of an optically thin body as a function of the angle of view. A dense point in the body is translated into a sinusoidal trajectory in the sinogram, hence its name. Many techniques are available to restore the $2 \mathrm{D}$ density map of the section from the sinogram, but all of them require an unchanging body during the acquisition time.

The sinogram concept can be applied to solar plumes even if it can not be used for the restoration. Assuming a unique dense column of electrons as an ideal model of electronic density on the coronal hole, its section in the plane of sight will be a bright point. In the sinogram this point will draw a sinusoidal trajectory due to the rotation of the Sun. Changes in the electron density during the rotation time translates into the point brightness as well as changes in the intensity of the trajectory. Even if the lifetime of a bright point is small it is possible to deduce their position in the coronal hole plane from their short trajectory trace in the sinogram. The frequency of any trajectories is defined by the Sun rotation rate, its zero axis coincides with the solar axis, there are only two free parameters: amplitude and phase (see Fig. 2). Two points are enough to determine such parameters. Precision depends on the position error and on their relative distance. Because the intensity of sources change in our sinogram, we call it the "time intensity diagram" or TID.

To study the electron density map of the polar regions we applied the TID concept to successive images of LASCO-C2. We used the sequence of 402 LASCO-C2 images obtained from $1997 / 03 / 21$ at $22 \mathrm{~h} 10 \mathrm{~m} 35 \mathrm{~s}$ to $1997 / 03 / 24$ at $16 \mathrm{~h} 22 \mathrm{~m} 41 \mathrm{~s}$, that is 2 days and 18 hours of continuous observations with a mean interval between frames of 9.9 minutes. This sequence was observed for the special purpose of plume analysis. Long exposures get the best possible S/N (signal to noise) ratio.

In the original images plumes appear like rays of dim contrast (of $2 \sim 5 \%$ ) over a high background due to the F-corona. This is the light generated by the zodiacal dust cloud around the Sun. A specific processing pipeline was applied including:

1. photometric equalization;

2. subtraction of the F-corona, straylight, impacts of cosmic particules, stars;

3. definition of the divergence point (apparent center of the super-radial expansion);

4. denoising; 
The divergence point has been found by using the Hough transform. The set of rays emerging from this (invisible) common point is converted into a set of aligned points in the parameter space. Once the coordinates of this point are known, the directional denoising procedure is applied to each ray. The straight line which fits this set of points is, in this space, the translation of the divergence point. A low degree log-log regression along each radial direction is a very efficient method. A circular profile centered on the divergence point is extracted from each filtered image of the sequence; the set of successive profiles defines the TID (see Fig. 3) which shows the profile brightness versus the angle (horizontal axis) and versus the time (vertical axis).

A large set of entangled trajectories can be observed in the TID showing a fuzzy appearance and an increasing and decreasing brightness (Fig. 3). As said before, short trajectories are enough to fix the center of plumes in the plane over the Sun pole with good approximation. Such paths depend on two parameters: phase and amplitude. Therefore it is possible to define them by fitting a tangent line to segments of trajectories. This method produces meaningful results when the rotation of the Sun is the predominant cause of the plume changes.

Studying the temporal evolution of polar plumes using the TID, we have shown (Llebaria et al. 1998) that WL plumes are enduring and recurrent structures in rigid rotation with the solar magnetic field. The time-scale of blinking can be as short as 2 hours. Observing differences in brightness along a trajectory we found a mean radial flow speed of $460 \mathrm{~km} / \mathrm{s}$ in the range 3.0-7.0 $\mathrm{R}_{\odot}$ (Llebaria et al, 2002). Furthermore, the time derivative of the sinogram revealed the existence of a set of intense, short-lived bursts lasting 1 to 2 hours and superimposed on the background due to the long-term activity of plumes.

\section{GEOMETRIC ANALYSIS}

The main assumption in the preceding approach is the existence of physical plumes filling the coronal hole. But this raises a set of basic questions: Do these plumes show well defined boundaries? Are they isolated structures such as magnetic tubes of enhanced electron density? Or do they result from geometrical projection effects of line-of-sight integration through plasma slabs or curtain folds? Are we able to discover their geometric parameters from the projections? Moreover, is it possible to distinguish between plume and interplume, to define what a plume is in the original image?

As no clear response emerged from the visual analysis of profiles, we obtained the spectra and the autocorrelation function of different profiles. The determination of the geometric and photometric parameters of a "standard" plume was the main objective. In fact we aimed to determine "typical diameters" by using classical auto-correlation methods. These methods need an initial step to remove the trend generated by the lowest frequencies (i.e., a background subtraction); in our case the correlation length which defines the "typical diameter" depends strongly on the cutoff frequency used for the detrend operation. This fact strongly supports the idea that the "typical diameter" lacks any physical meaning.

The spectrum (Fig. 4) revealed a $1 /|f|^{n}$ decay ( $f$ stands for the spatial frequency) as an unambiguous signature of the fractal nature of these profiles. More precisely the behavior points to the $1 / f$ family of fractal processes (Llebaria et al. 2002). This family includes a large set of self-affine fractals whose empirical power spectra are of the form $S(f)=\sigma^{2} /|f|^{\gamma}$, where $\gamma$ is a parameter in the range $0.5<\gamma<3$. We recall that $D=(5-\gamma) / 2$ where $D$ is the fractal dimension introduced by Mandelbrot and Van Ness (1968) to describe in a rigorous and quantitative sense the roughness of geometrical objets. For $1 \mathrm{D}$ Euclidean profiles, a small $D \simeq 1$ is associated with smooth variations and a large $D \simeq 2$ with rough ones. Roughly speaking, in contrast with a pure Gaussian noise, in such profiles the lower the frequency, the larger the relative weight. The weight increases as frequency decreases following an exponential law.

Plume profiles extracted from the TID show the uniform negative slopes of $2 \ldots 2.5$ on the loglog reference plane. This is characteristic of the fractal profiles of dimension $1.5 \ldots 1.3$. The initial result from the "power-spectrum" method has been confirmed by using other classical approaches 


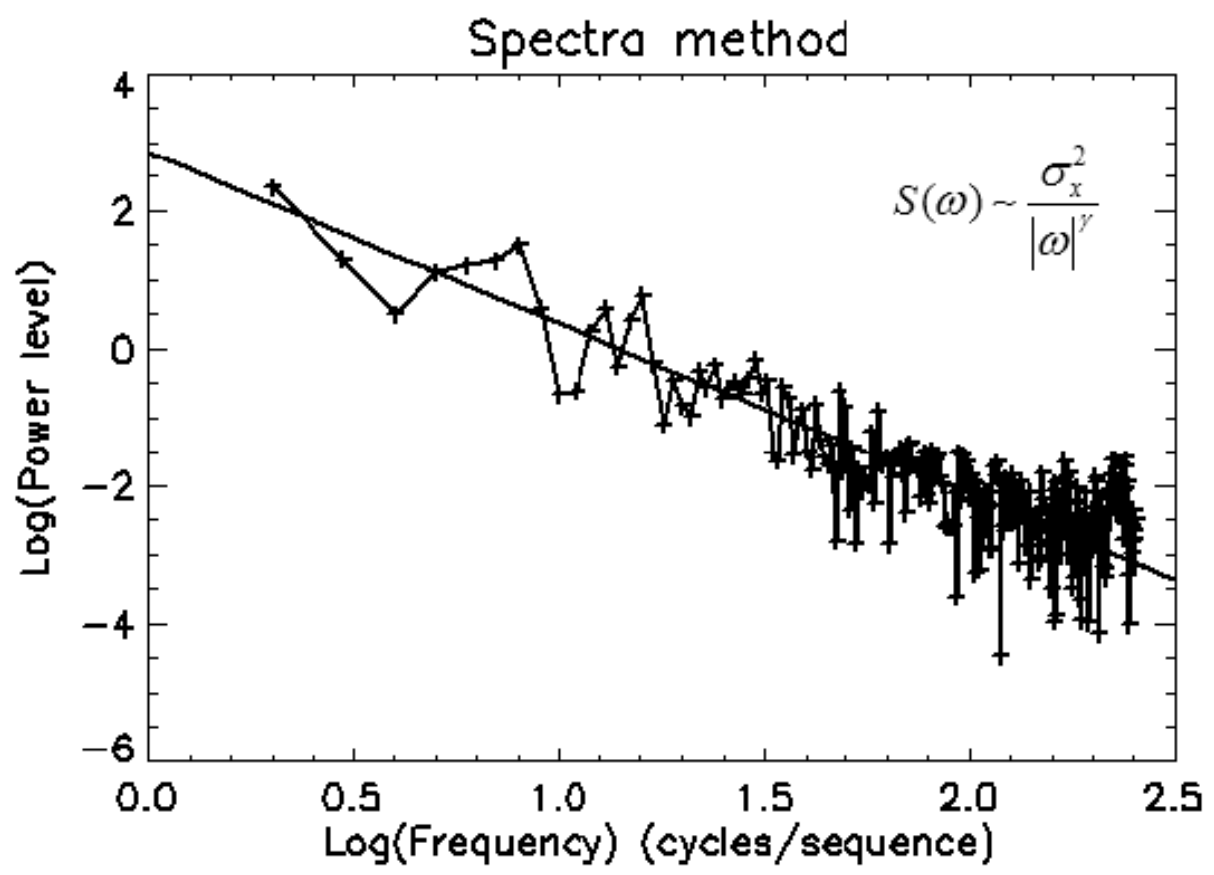

FIGURE 4: Fractal dimension $D$ estimated for the profile no. 210 of the TID. $D$ is deduced from the $\gamma$ coefficient $D=(5-\gamma) / 2$.

to fractal analysis such as the "box-counting" method, the "difference statistics" method (or "correlation", or "variance" method) and the "covering-blanket" method etc. The fractal distribution of electronic densities on the surface near the coronal hole explain the failure to define specific plumes as "objets" with precise positions and "typical diameters" even if we observe the dramatic radial coherence of the observable texture between 3 and 7 solar radii. The multiscale analysis seems to be more appropriate in order to redefine this classical view in a new sense.

\section{MULTIRESOLUTION ANALYSIS}

The multiresolution analysis, MRA, was first applied to determine the distribution of angular orientations of plumes. Amazingly, i) there is only a limited range of orientations, a result which implies a strong selection effect relative to the plane of the sky, and ii) this set of orientations is centered around the plane of the sky with a $7^{\circ}$ inclination angle relative to the Sun polar axis (due to the SOHO position on this date). The MRA was subsequently applied, profile by profile, in the direction orthogonal to the time coordinate (see Fig. 6). Our objective was here to determine a relationship between the spatial scale of plumes and its duration. Its mean lifetime varies from 1 to 20 hours as angular size varies from 0.2 to 10 degrees (see Fig. 7).

\section{DYNAMIC FORWARD MODELING}

To verify the hypothesis of fractal 2D distribution on the coronal hole space we carried out the simulation of the full measure process. Such simulation includes, for each image shot: 1) the fractal 2D distribution, 2) its projection in the 3D space of the coronal hole, 3) conversion to brightness in the observer direction (Thompson scattering), 4) integration along the line of sight, 5 ) instrument effects. As the TID shows, the evolution of plumes with time is due to geometric effects as the solar corona rotates and to fractal evolution of the electron density distribution. Electrons in the solar corona scatters the Sun light by the Thomson effect: the radiance pattern is anisotropic and polarized. The final image results from the addition of the local contributions 


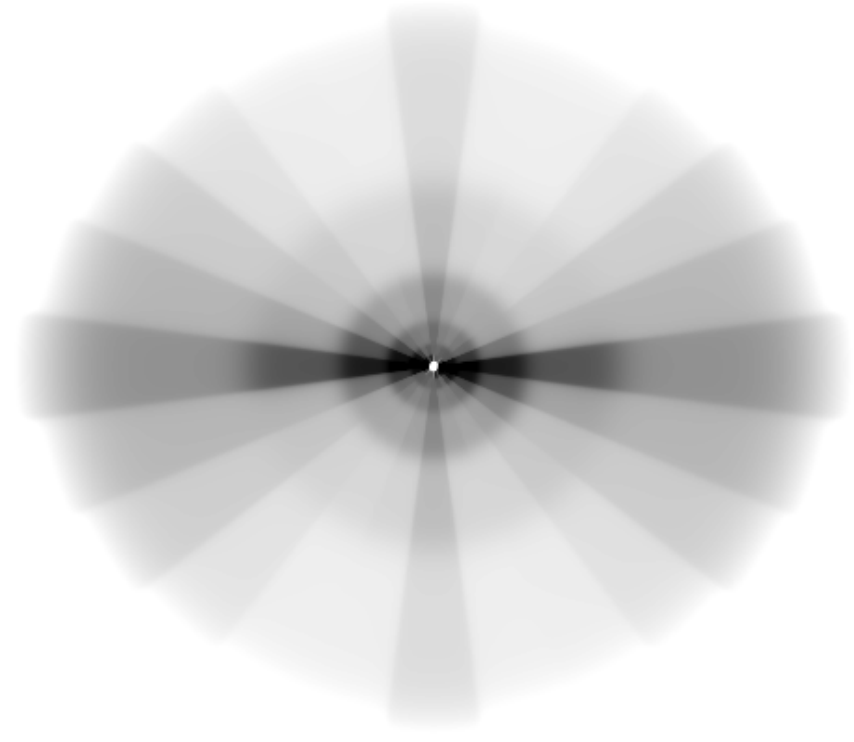

FIGURE 5: Spectral power pattern for the TID in the Fourier space. $w=0$ is in the center. The intensity is in logarithmic scale (from white to black). Image shows the strong predominance of low frequency components. Most of the intermediate frequency (IF) and high frequency (HF) components are in the $-22.5+7.5$ range.

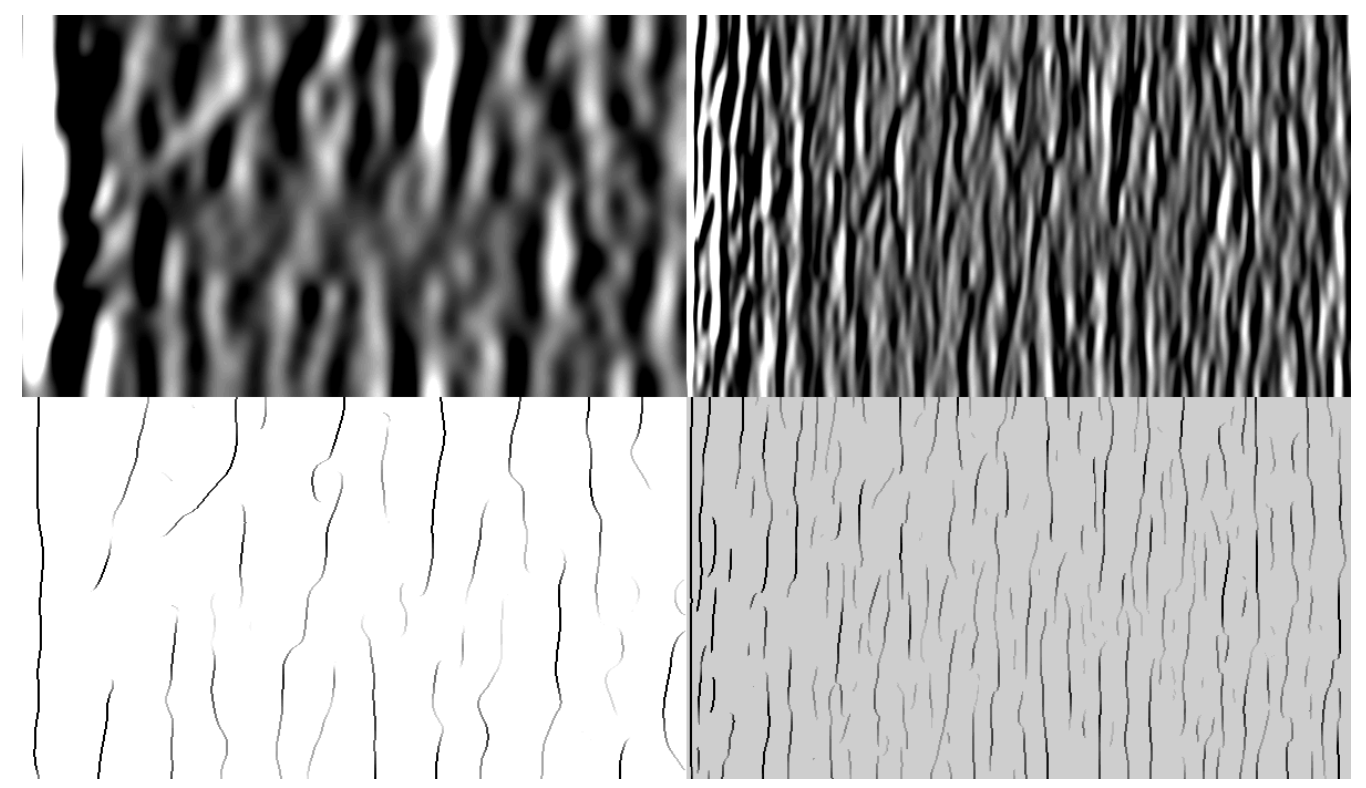

FIGURE 6: TID was analyzed row by row by using a Morlet wavelet in progressively wide scales. There is a large spectrum of sizes, from the resolution scale used to a few degrees. Represented here are two of them, the sine component at top and plume ridges at bottom. Ridges retrace the evolution of the centers of each structure at each scale. The scales represented here are 2 and 8 deg of polar angle from top to bottom and the time interval is 66 hours. The ridge distribution is used as the basis for the statistical analysis. 

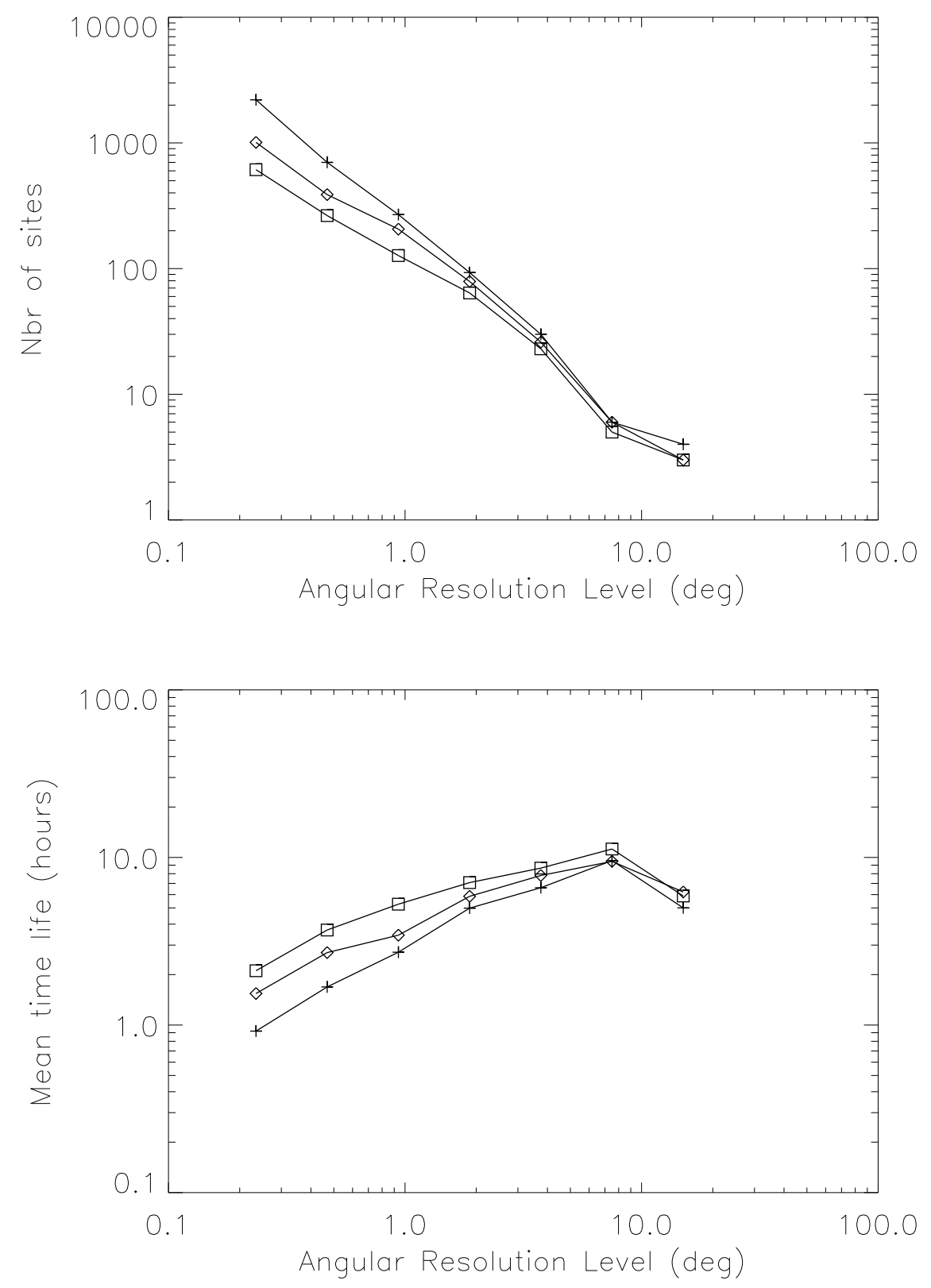

FIGURE 7: In the upper graph the number total of plume structures as function of angular size is given for different low pass pre-filterings in the $t$ coordinate direction (15, 30 and 50 min of time). In the lower graph the mean lifetime of structures of each level is given for the same set of pre-filterings. A selection effect due to limits of time interval as well as insufficient statistics can be can be observed at 16 deg of resolution. 
of scattered light in the line of sight defined by each pixel. To build the sequence of images and subsequent simulated TID we use two different methods:

- Fractal affine model: In this model the initial fractal distribution is defined in a 2D+1D space of frequencies, then converted to direct space (X,Y and time). In short:

$$
\widehat{S}\left(f_{x}, f_{y}, f_{t}\right)=\frac{X_{\lambda}\left(f_{x}, f_{y}, f_{t}\right) e^{i \Phi\left(f_{x}, f_{y}, f_{t}\right)}}{\|f\|^{\gamma}}
$$

where $X_{\lambda}$ and $\Phi$ are uniform random arrays and $f_{x}, f_{y}, f_{t}$ are the coordinates of the space of frequencies.

- Evolving multiscale model: In this model (see Fig. 8) the fractal distribution is built octave ${ }^{1}$ by octave in a $2 \mathrm{D}$ space of frequencies then translated to direct image space. From an image to the next one, each octave evolves at its own rate, fast for the detail scale, slow for broad scales. The sum of octaves defines a distribution which will be projected once the corresponding rotation has been applied. Let $A_{i}$ be a set of $I$ independent fractal images and $B_{k i}$ the associated collection of octaves: for each index $i$ there is a subset of $K$ passband images (octaves). The index $k$ defines the resolution level, $k=0$ the highest and $k=K-1$ the lowest one. Let $\Delta t$ be the time interval between $A_{i}$ and $A_{i+1}$, the definition of an image $S(t)$ of electron density distribution at the time $t$ will be:

$$
S(x, y ; t)=\sum_{k=0}^{k=K-1} \sum_{i=1}^{i=I} f_{k i, i}(t-k i \Delta t) B_{k, i}(x, y)
$$

Each image $S(x, y ; t)$ for $t \in t_{1}, t_{2}, t_{3}, \ldots t_{l}$ will be rotated afterwards to build the simulated sequence. Notice that $t_{i+1}-t_{i}>>t$. The weight functions $f_{k i, i}(t-k i \Delta t)$ form a double set (in $k$ and $i$ ) of scaled and translated functions corresponding to each scale $k$ and time interval $k . \Delta t$. They must be normalized, it is $\sum_{i=1}^{i=I} f_{k i, i}=1$. Such functions (they are splines) allow for a smooth transition between successive frames and between different resolutions.

In practice, the latter method can be more easily handled in their algorithmic translation than the former; because it requires less computer resources than the former one it allows for a large parameter excursion. Final images (see Fig. 11) show a set of simulated TID for different levels of initial $D$. To obtain a visual appearance and fractal dimension in the final TID similar to the original sequence we needed an initial $D=2.9$ in the plane of sight. Careful testing of lacunarity and multifractal spectra from the original sequence and from simulations are currently in progress.

\section{CONCLUSIONS}

We have found that white light polar plumes show a clear fractal structure, characterized by a fractal dimension $D=1.5 \pm 0.2$. We assume that the $2 \mathrm{D}$ fractal distribution of the electron density in sections of coronal holes is the origin. Multiresolution analysis has shown that there is an overlap of fuzzy structures. We have observed that the larger the size of these structures, the larger its lifetime. This picture deeply modifies the classical view of plumes as "tubes or sheets of plasma". These structures are statistically self-similar in sizes embracing scales from a few degrees up to the LASCO/C2 resolution scale $\left(<10^{\prime}\right)$. Probably irregular patches of dense plasma are surrounded by or mixed with low density regions with fuzzy transitions between them. It seems that LASCO-C2 is blind to the actual low resolution limit of these structures. The fibrous structure of coronal holes remains as an open question, but the fractal 2D distribution of the observed macro-structures appears highly compatible with this assumption. Simulations are a powerful tool to constrain models of 2D distribution of the electron density.

\section{REFERENCES}

[1] Deforest C.E., 1998, ESP SP-421, 63

\footnotetext{
${ }^{1}$ Octaves correspond to the set of resolution levels
} 


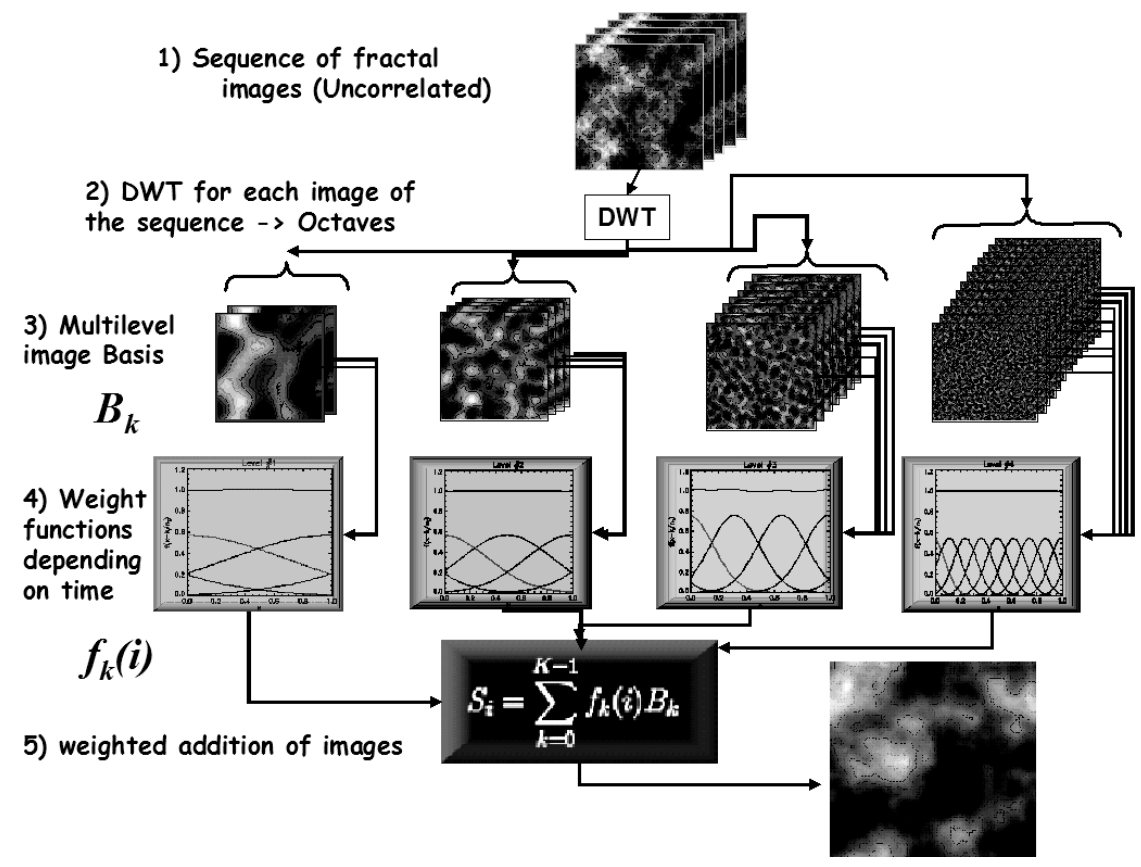

FIGURE 8: Evolving multiscale model. The dynamic sequence is built up as 2D spatial $+1 \mathrm{D}$ temporal volume of successive 2D frames Each volume section is a map of electron density over the reference surface. The fractal distribution is built octave by octave in a 2D space of frequencies, then translated to direct image space. From an image to the next one each octave evolves at different rates, fast for the detail scale, slow for broad scales. The sum of octaves defines a distribution which will be projected once the corresponding rotation has been applied. See the text for a more extended explanation.

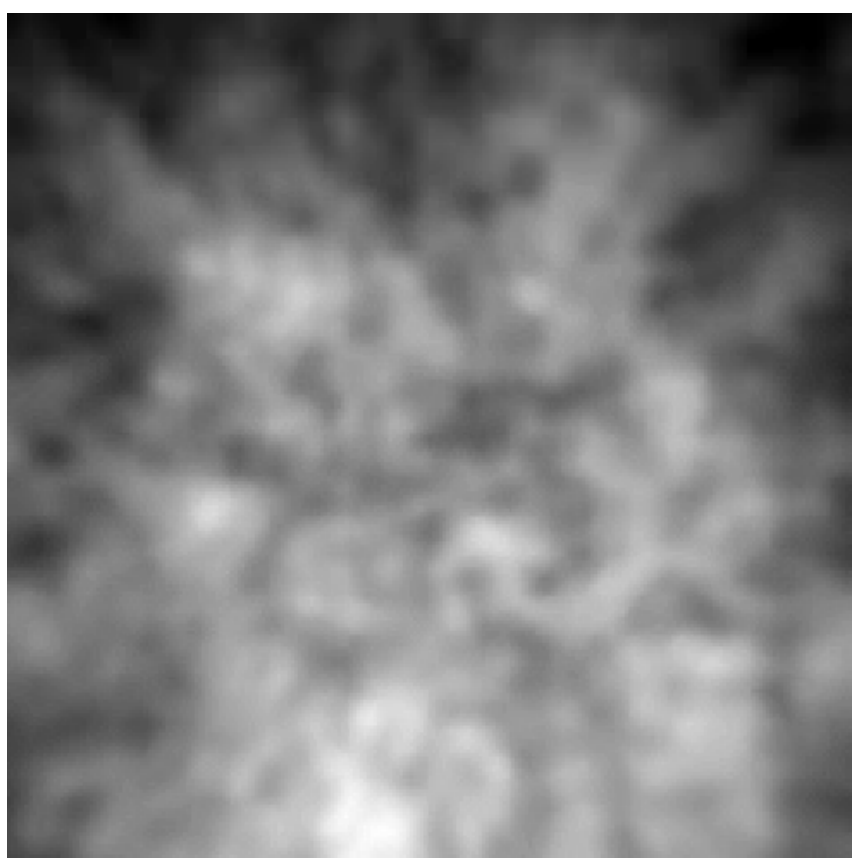

FIGURE 9: 2D electron density model in a plane of sight over the coronal hole 


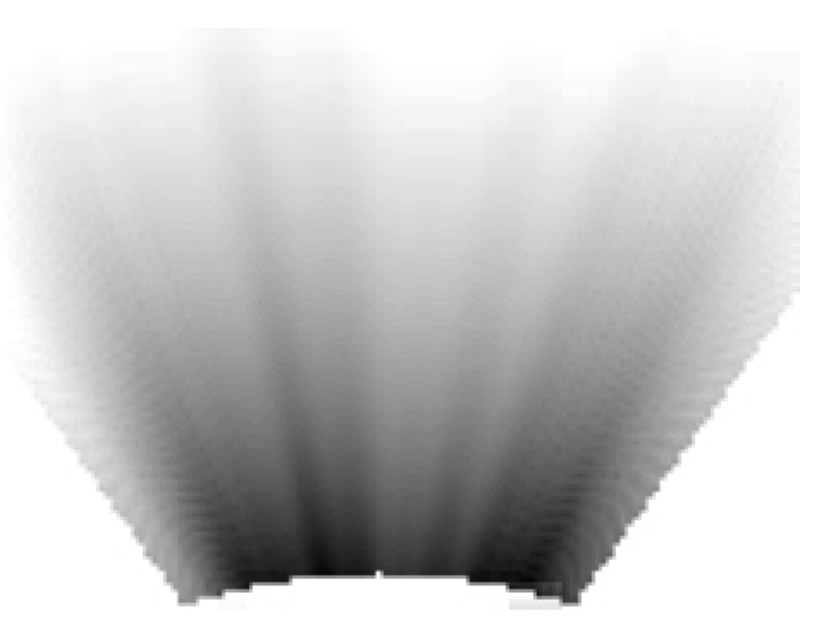

FIGURE 10: Projection of simulated plumes in the plane of view. Plumes are in black (negative like).

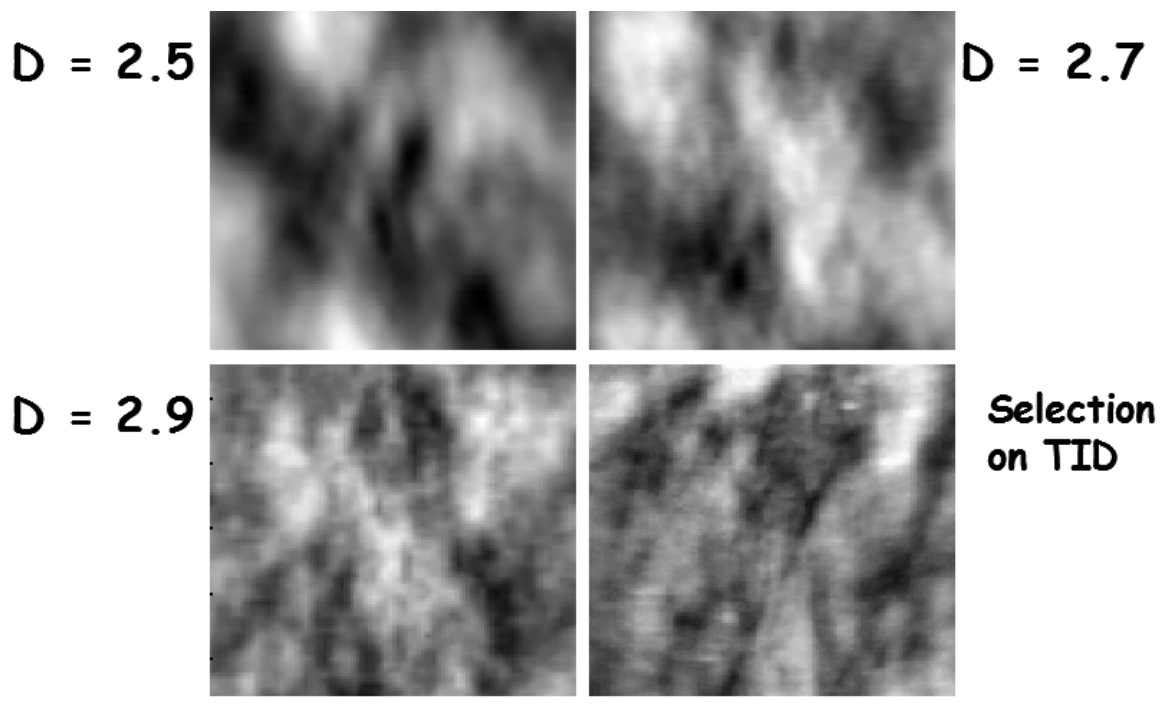

FIGURE 11: Simulated TIDs found for different initial fractal dimensions in the 2D plane. Dimension (D) is the parameter defining the fractal 2D field in the coronal hole. To reach a $1 \mathrm{D}$ profile dimension of 1.5 by projection requires a $2 \mathrm{D}$ fractal dimension of 2.9 . 
[2] Deforest C.E., Lamy P., Llebaria A., 2001, ApJ 560, 490

[3] Lamy P., Llebaria A., Koutchmy S. et al., 1997, SOHO V, ESP SP-404, 487

[4] Llebaria A., Lamy P., Deforest C., Koutchmy S., 1998, ESP SP-421, 87

[5] Llebaria A., Thernisien A., Lamy P., 2002, Adv. Space Res. 29, 3, 343

[6] Mandelbrot B.B. and Van Ness J.W., 1968, SIAM Rev. 10, 4, 422

[7] Wornell G.W. and Oppenheim A.V., 1992, IEEE Trans. Signal Processing 40, 611

[8] Llebaria A., Saez F., Lamy P., 2002, SOHO 11, ESP SP-508, 391 